\title{
Calculating vibrational mode contributions to sound absorption in excitable gas mixtures by decomposing multi-relaxation absorption
}

\section{spectroscopy}

Kesheng Zhang ${ }^{\mathrm{a}, \mathrm{b}}$, Yi Ding ${ }^{\mathrm{c}}$, Ming Zhu ${ }^{\mathrm{c}, *}$, Mingzhe $\mathrm{Hu}^{\mathrm{d}}$, Shu Wang ${ }^{\mathrm{c}}$, Yingqun Xiao ${ }^{\mathrm{a}}$

a Guizhou Key Laboratory of Electric Power Big Data, Guizhou Institute of Technology, Guiyang 550003, Guizhou, China

b School of Information Engineering, Guizhou Institute of Technology, Guiyang 550003, Guizhou, China

c School of Electronic Information and Communications, Huazhong University of Science and Technology, Wuhan 430074, Hubei, China

d Department of Physics and Electronics, Liupanshui Normal University, Liupanshui, Guizhou, 553004, China

\section{ABSTRACT}

Sound relaxational absorption spectroscopy of excitable gas mixtures is potentially applied for gas composition detection. The relaxation of vibrational modes of gas molecules determines the sound relaxational absorption. However, to our knowledge, the contribution of each vibrational mode available in gas mixtures to sound multi-relaxation absorption has not been calculated in existing literature. In this paper, based on the decoupled expression of the effective isochoric molar heat for a gas mixture, a sound multi-relaxation absorption spectrum is decomposed into the sum of single-relaxation spectra. From this decomposable characteristic, the contribution of each vibrational mode available in the gaseous medium to the multi-relaxation absorption is obtained at room temperature. For various gas compositions including carbon dioxide, methane, nitrogen etc., the calculated contributions of vibrational modes are verified by the comparison with experiment data. We prove the following views with quantifiable outcomes that the primary molecular relaxation process associated with the lowest mode plays the major role in acoustic relaxational absorption of gas mixtures; the mode with lower vibrational frequency provides higher contribution to the primary relaxation process. This work could provide a deeper insight into the relationship between the sound relaxational absorption spectroscopy and gas molecules.

Keywords: sound absorption; Molecular vibrational relaxation; Mode contributions; Decomposing multi-relaxation absorption spectra

* Corresponding author.

E-mail: zhuming@mail.hust.edu.cn 


\section{Introduction}

Acoustic relaxational absorption spectroscopy of gas mixtures is potentially applied for gas composition detection, such as practical applications for real-time monitoring of natural gas [1-8]. The propagation of acoustic waves in gases is fundamentally a molecular phenomenon [9]. The sound absorption in excitable gases (polyatomic or diatomic gases or their mixtures) is caused by two mechanisms: the classical absorption associated with transport phenomena (e.g., heat conduction and viscosity) and the non-classical, or relaxational absorption associated with the lag in adjustment between the internal states of the molecules and their external energy [10]. In a thermal equilibrium, the energy distribution of a gas between the external (translational) molecular kinetic energy and the internal (e.g., vibrational, rotational) states of energy of the molecules only depends on the temperature of the gas. When the equilibrium is disturbed by the propagation of an acoustic wave through the gas, there is an instantaneous increase (due to compression) in the external energy. By molecular collisions, some of this energy increase enters into the internal states. A certain relaxation time (or time lag) is required for this energy to be transferred back to the external form to reestablish equilibrium. As a result of that, much of this energy released on de-excitation will be released as heat to give rise to a net absorption of acoustic energy - the relaxational absorption. For most simple molecules at room temperature, the rapid equilibration between rotation and translation makes Vibrational-Rotational plus Rotational-Translational energy transfer process indistinguishable from the Vibrational-Translational (V-T) process. Thus, rotation is usually classified as translation as "external" degrees of freedom (DOF) to distinguish from vibration as the "internal" DOF which takes a longer time to adjust [10]. Thus, the internal DOF thermal relaxation is almost entirely attributed to the vibrational modes, whose relaxation processes are the cause for the sound relaxational absorption around room temperature [3].

Herzfeld and Rice made the first application of a vibrational relaxation process to the theoretical calculation of acoustic absorption [11]. Landau and Teller treated the vibrational energy as the multileveled system of a harmonic oscillator to calculate V-T relaxation time [12]. Schwartz, Slawsky, and Herzfeld (SSH) refined the Landau and Teller's theory by including a one-for-one quantum exchange of vibrationalvibrational $(\mathrm{V}-\mathrm{V})$ energy transfer in two diatomic mixtures [13]. Tanczos developed the SSH theory to calculate the absorption and dispersion of acoustic wave in pure polyatomic gases allowing for the two quanta for one quantum V-V exchange [14]. Dain, Lueptow (DL) and Petculescu predicted the sound relaxational absorption in 
ternary mixtures with the extended SSH relaxation equations, and attempted to describe the degree of participation of a vibrational mode in the acoustic relaxation by using normalized eigenvectors of matrix $\mathbf{A}[15,16]$. Recently, we presented the models to predict sound relaxational absorption spectra in multi-component gas mixtures [17] and decoupled the extended SSH energy relaxation equations to explain how a multimode relaxation leads to the peaks in the corresponding sound absorption spectrum [18]. We also developed an algorithm [4] to capture the primary vibrational relaxation processes from the acoustic measurements at two operating frequencies and applied the algorithm into gas composition detection by employing the absorption spectral peak locations and effective relaxation area (ERA) of spectral peaks in gas mixtures [6-8]. Generally, an excitable gas contains two or more molecular vibrational modes to arouse a multi-relaxation process which is synthesized by the complicated strong vibrational-vibrational (V-V) energy coupling [19,20]. As far as we know, in excitable gas mixtures, the contribution of each vibrational mode to acoustic multi-relaxation absorption has not been calculated or measured in existing literature. Therefore, it is still desirable to obtain this straightforward relation between vibrational modes and acoustic absorption.

In this paper, based on the decoupled effective isochoric molar heat (IMH) derived from the extended Tanczos relaxation equations, we demonstrate that the sound relaxational absorption spectrum due to a multi-relaxation process can be decomposed into the sum of spectra ascribed to a set of single-relaxation processes which belong to the multi-relaxation process (In our previous works [17,18], we directly calculated the multi-relaxation absorption spectrum, while we will obtain the multi-relaxation absorption spectrum by the sum of its single-relaxation absorption spectra in this paper). This decomposition means that the acoustic absorption in excitable gases not only can be traditionally divided into the classical absorption part and the relaxational absorption part, the relaxational absorption part under a multi-relaxation process can be further decomposed into the sum of single-relaxation processes spectra. From this decomposable characteristic of multi-relaxation absorption, we can calculate the contribution of each vibrational mode available in the gaseous medium to the sound relaxational absorption. The quantitative relation between the molecular makeup of gas mixtures and acoustic absorption revealed in this paper will improve our understanding in the practical applications of gas detection with acoustic absorption spectroscopy.

This paper is organized as follows. In Section 2, the vibrational relaxation 
equations given by Tanczos [14] have been extended to multi-component gas mixtures. Apart of that, based on the decoupled expression of the effective IMH, we demonstrate that an acoustic relaxation process aroused by multiple vibrational modes is the sum of single-relaxation processes with different relaxation times and strengths, showing a multi-modes process is a multi-relaxation process that can be expressed in terms of the process IMHs and the relaxation times of the single-relaxation processes. Section 3 demonstrates a sound multi-relaxation absorption spectrum can be decomposed into the sum of single-relaxation absorption spectra, and thus we can calculate the vibrational mode contributions to the acoustic relaxational absorption. In Section 4, we compare the calculations of decomposed sound absorption spectra and vibrational mode contributions with the experimental data to validate the proposed model. Section 5 concludes this paper.

\section{Decoupled effective isochoric molar heat of gas mixture}

\subsection{Extended Tanczos relaxation equations}

In our previous work $[17,18]$, we have applied the SSH coupled relaxation equations, which only allows for a one-for-one quantum exchange of $\mathrm{V}-\mathrm{V}$ energy transfer, into a multi-component gas mixture. Similarly, for a gas mixture with $W$ kinds of molecules including $N$ vibrational modes, we can extend the Tanczos relaxation equations [14], which permit two quanta for one quantum $\mathrm{V}-\mathrm{V}$ exchange, to a general form as

$$
\begin{aligned}
\frac{\mathrm{d} \Delta T_{j}^{\mathrm{vib}}}{\mathrm{d} t}= & \left(\Delta T-\Delta T_{j}^{\mathrm{vib}}\right) k_{j j}-\sum_{\substack{k=1 \\
k \neq j}}^{N}\left(\Delta T-\Delta T_{k}^{\mathrm{vib}}\right) k_{j k}, j, k=1, \ldots, N, j \neq k . \\
k_{j j}= & k_{10}(j)+\sum_{\substack{k=1 \\
k \neq j}}^{N}\left[k_{01}^{10}(j, k)+2 \exp \left(-h v_{j} / k_{B} T_{0}\right) k_{01}^{20}(j, k)+\theta_{k}^{-1} k_{02}^{10}(j, k)\right] \theta_{k}^{-1} \theta_{j} g_{k} \\
& \left.k_{j k}=k_{01}^{10}(j, k)+2 \exp \left(-h v_{j} / k_{B} T_{0}\right) \theta_{j}^{-1} k_{01}^{20}(j, k)+k_{02}^{10}(j, k)\right] v_{j}^{-1} v_{k} \theta_{k}^{-1} \theta_{j} g_{k} .
\end{aligned}
$$

where $h$ is Planck's constant, $k_{B}$ is Boltzmann's constant, $g_{k}$ is the degeneracy of mode $k . \theta_{j}=1-\exp \left(-h v_{j} / k_{B} T_{o}\right)$ is the characteristic temperature of vibrational mode $j, T_{0}$ is the equilibrium temperature, $v_{j}$ and $v_{k}$ are the characteristic frequencies of mode $j$ and mode $k$ respectively. $\Delta T=T-T_{0}$ and $\Delta T_{j}^{\mathrm{vib}}=T_{j}^{\mathrm{vib}}-T_{0}$ are the fluctuations of the translational and vibrational temperature respectively, $T$ is the instantaneous temperature of external DOF, $T_{j}^{\mathrm{vib}}$ is the instantaneous temperature of vibrational mode $j$. The V-T transition rate $k_{10}(j)$ is the number of transitions per second per molecule in which the 
energy goes from the first-excited-vibrational level into translational motion, i.e. the state of mode $j$ goes from $1 \rightarrow 0$ and the vibrational level of molecule $l$ is unaltered; the $\mathrm{V}-\mathrm{V}$ transition rate $k_{01}^{10}(j, k)$ is the rate when mode $j$ goes from $1 \rightarrow 0$ and mode $k$ goes from $0 \rightarrow 1[18,21]$. The $\mathrm{V}-\mathrm{V}$ transition rates $k_{01}^{20}(j, k)$ and $k_{02}^{10}(j, k)$ are the energy transition rates involving a two-for-one interchange of quantum

$$
k_{01}^{20}(j, k)=a_{k} Z(j, k) \times P_{0-1(k)}^{2-0(j)}, \quad k_{02}^{10}(j, k)=a_{k} Z(j, k) \times P_{0-2(k)}^{1-0(j)} .
$$

Here, $a_{k}$ is the mole fraction of mode $k, Z(j, k)$ is the hard sphere collision rate between the molecules having the mode $j$ and $k$ [21]. $P_{0-0(l)}^{1-0(j)}$ is the V-T transition probability (i.e. the probability that the state of mode $j$ goes from $1 \rightarrow 0$ and the vibrational level of molecule $l$ is unchanged). $P_{0-1(k)}^{1-0(j)}$ is the V-V transition probability that during a binary collision the mode $j$ of one molecule will change its quantum state from 1 to 0 , while simultaneously a second mode $k$ in the other molecule will change its state from 0 to $1 . P_{0-1(k)}^{2-0(j)}$ and $P_{0-2(k)}^{1-0(j)}$ are the $\mathrm{V}-\mathrm{V}$ transition probabilities involving a two-for-one interchange of quantum. The calculation expressions for those transition probabilities are given by Tanczos [14,16,21]. Table 1 in ref. [18] provides all the necessary parameters to calculate the vibrational transition probabilities for nitrogen, oxygen, chlorine, methane, and carbon dioxide.

\subsection{Decoupled effective isochoric molar heat}

The effective IMH represents the macroscopic "footprint" of vibrational relaxation effects [3]. For the relaxing mixture, its effective IMH $C_{V}^{\text {eff }}$ is [18]

$$
C_{V}^{\mathrm{eff}}=C_{V}^{\infty}+\sum_{j=1}^{N} a_{j} C_{j}^{\mathrm{vib}} \mathrm{d} T_{j}^{\mathrm{vib}} / \mathrm{d} T .
$$

where $C_{V}^{\infty}=\sum_{l=1}^{W} a_{l} C_{l}^{\infty}$ is the external IMH of the mixture or the non-relaxation part of heat capacity, $a_{l}$ is the mole fraction of molecule $l\left(\sum_{l=1}^{W} a_{l}=1\right), C_{l}^{\infty}$ is the external IMH of molecule $l$. For a linear molecule, there are 3 translations and 2 rotations, so $C_{l}^{\infty}=5 R / 2$ ( $R$ is the universal gas constant); for a nonlinear molecule, $C_{l}^{\infty}=3 R$. $C_{j}^{\mathrm{vib}}$ is the vibrational IMH of mode $j$ calculated by the Planck-Einstein function [15], $a_{j}$ is the mole fraction of mode $j, \mathrm{~d} T_{j}^{\mathrm{vib}} / \mathrm{d} T$ is the ratio of vibrational-to-acoustic temperature fluctuations of mode $j$.

To calculate $\mathrm{d} T_{j}^{\mathrm{vib}} / \mathrm{d} T$ in Eq. (3), we need convert the relaxation equations into its algebraic form. In an acoustic field with small amplitude of harmonic plane wave of angular frequency $\omega$, Eq. (1) becomes the following algebraic relaxation equations [18] 


$$
\begin{gathered}
y_{j}\left(k_{j j}+\mathrm{i} \omega\right)-\sum_{\substack{k=1 \\
k \neq j}}^{N} y_{k} k_{j k}=k_{j j}-\sum_{\substack{k=1 \\
k \neq j}}^{N} k_{j k}, \\
y_{j}=\Delta T_{j}^{\mathrm{vib}} / \Delta T \cong \mathrm{d} T_{j}^{\mathrm{vib}} / \mathrm{d} T, \quad j, k=1, \ldots, N, j \neq k .
\end{gathered}
$$

Then, Eq. (4) can be expressed in a matrix form

$$
(\mathrm{i} \omega \boldsymbol{I}+\boldsymbol{R}) \mathbf{y}=\boldsymbol{H},
$$

where $\mathbf{y}_{j}=y_{j}, \quad \boldsymbol{R}_{j j}=k_{j j}, \quad \boldsymbol{R}_{j k}=-k_{j k}, \quad \boldsymbol{H}_{j}=k_{j j}-\sum_{k=1, k \neq j}^{N} k_{j k}, j, k=1, \ldots, N, j \neq k ; \quad \boldsymbol{I}$ is the identity matrix. Eq. (5) links the temperature fluctuations of the vibrational mode and external DOF. If the energy-transition-rate matrix $\boldsymbol{R}$ can be diagonalized in the form as $\boldsymbol{R}=\boldsymbol{V} \boldsymbol{\Lambda} \boldsymbol{V}^{-1}$, from Eq.(5), we have [18]

$$
y_{j}=\sum_{n=1}^{N} \frac{V_{j n} h_{n}^{\prime}}{\left(1+\mathrm{i} \omega \lambda_{n}^{-1}\right)}, j=1,2, \mathrm{~K}, N .
$$

Where $V_{j n}$ is the entry of matrix $\boldsymbol{V}, \lambda_{j}$ is the eigenvalue of $\boldsymbol{R}$, $h_{n}^{\prime}=\lambda_{n}^{-1} \sum_{k=1}^{N} V_{n k}^{\prime} h_{k} \quad\left(\lambda_{n}^{-1}\right.$ is the reciprocal of eigenvalue of $\boldsymbol{R}, V_{n k}^{\prime}$ and $h_{k}$ are the entries of matrix $\boldsymbol{V}^{-1}$ and $\boldsymbol{H}_{j}$ respectively).

Noting that $y_{j} \cong \mathrm{d} T_{j}^{\mathrm{vib}} / \mathrm{d} T$ and substituting Eq. (6) into Eq. (3), we obtain the decoupled expression of the effective IMH for the mixture as

$$
C_{V}^{\mathrm{eff}}=C_{V}^{\infty}+\sum_{n=1}^{N} \frac{h_{n}^{\prime} \sum_{j=1}^{N} a_{j} C_{j}^{\mathrm{vib}} V_{j n}}{\left(1+\mathrm{i} \omega \lambda_{n}^{-1}\right)}=C_{V}^{\infty}+\sum_{n=1}^{N} \frac{C_{n}^{*}}{\left(1+\mathrm{i} \omega \tau_{n}\right)}, n=1,2, \mathrm{~L}, N .
$$

Where $C_{n}^{*}=h_{n}^{\prime} \sum_{j=1}^{N} a_{j} C_{j}^{\mathrm{vib}} V_{j n}$ and $\tau_{n}=\lambda_{n}^{-1}$, they are the process IMH and the isothermal relaxation time for one of decoupled single-relaxation processes respectively [18]. The effect of the delay in the transfer of energy between the internal and external DOF, i.e. the vibrational relaxation, is clearly displayed in the frequency dependence of $C_{V}^{\text {eff }}$. In Eq. (7), the equilibrium external and vibrational IMH are $C_{V}^{\infty}$ and $\sum_{n=1}^{N} C_{n}^{*}$ respectively. As the frequency of state alteration increases, an obvious decrease in the modulus of $C_{V}^{\text {eff }}$ will happen, making $C_{V}^{\text {eff }}$ ultimately reach the minimum limiting value $C_{V}^{\infty}$ when $\omega \rightarrow \infty$ (when the internal energy state is not populated). Inversely, the equilibrium thermodynamic heat capacity reaches the maximum limiting value $C_{V}^{\infty}+\sum_{n=1}^{N} C_{n}^{*}$ when $\omega \rightarrow 0$ (when the internal state is in equilibrium with the external states). At frequencies where the period of the compression-rarefaction cycle is comparable with the relaxation time, the 
frequency-dependent $C_{V}^{\text {eff }}$ leads to the sound relaxational absorption [22].

In Eq. (7), $N$ identifies the number of the decoupled single-relaxation processes, which is equivalent to the number of the vibrational modes as shown in Eq. (3). Actually, an excitable gas can be treated as a mixture of its DOF. Since every V-T transfer can provide a deexcitation path for the resident acoustic energy to the translational energy $[10,18]$, the number of the vibrational modes would be the same as the number of the single-relaxation processes. Therefore, a multimode relaxation process is a multi-relaxation process and it can be expressed in terms of the vibrational IMHs and the relaxation times of the single-relaxation processes.

Furthermore, the relation between $C_{V}^{\text {eff }}$ and the sound propagation characteristics is connected by the effective thermodynamic sound speed $c_{\mathrm{e}}=\sqrt{P_{0}\left(C_{V}^{\text {eff }}+R\right) /\left(\rho_{0} C_{V}^{\text {eff }}\right)} \quad\left(P_{0}\right.$ and $\rho_{0}$ are the equilibrium pressure and density respectively) [10]. From this relation, we obtained the sound multi-relaxation absorption spectrum $\alpha_{\mathrm{r}} \lambda=-2 \pi \cdot \operatorname{Im}\left(c_{\mathrm{e}}^{-1}\right) / \operatorname{Re}\left(c_{\mathrm{e}}^{-1}\right) \quad(\lambda$ is the sound wavelength) for the mixture in the previous works $[17,18]$.

\section{Vibrational mode contribution for sound absorption}

\subsection{Decomposition of sound multi-relaxation absorption spectrum}

The features of a sound absorption spectrum in excitable gases are determined by the characteristics of the resultant effective IMH [23]. Eq. (7) implies that there are exactly $N$ decoupled single-relaxation processes characterized by relaxation times $\tau_{n}$ $(n=1,2, \mathrm{~L}, N)$ and process IMHs $C_{n}^{*}(n=1,2, \mathrm{~L}, N)$ in the relaxing gas with $N$ vibrational modes. For those single-relaxation processes, the external IMH $C_{V}^{\infty}$ is the same; so the differences of absorption spectra due to those single-relaxation processes only result from their process IMHs and relaxation times.

From the measurements of acoustic absorption and speed at two frequencies, Petculescu and Lueptow (PL) propose a reconstructive algorithm [3] to synthesize the effective IMH of a single-relaxation process. Based on the PL algorithm, when considering a single-relaxation process with the relaxation time $\tau_{n}$ and the process IMH $C_{n}^{*}$, we can define the effective IMH of the single-relaxation process in the mixture as

$$
C_{n}^{\mathrm{eff}}=C_{V}^{\infty}+C_{n}^{*} /\left(1+\mathrm{i} \omega \tau_{n}\right),
$$

Acoustic propagation under the single-relaxation process of relaxing gas can be described in terms of its effective thermodynamic acoustic speed

$$
c_{n}^{e}=\sqrt{\left(P_{0} / \rho_{0}\right) /\left[\left(C_{n}^{\mathrm{eff}}+R\right) / C_{n}^{\mathrm{eff}}\right]},
$$

By using the relation between the effective speed $c_{n}^{e}$ and its effective wave number 
$k_{\mathrm{e}}$, i.e. $k_{\mathrm{e}}=\omega / c-\mathrm{i} \alpha_{n}=\omega / c_{n}^{e}$ ( $c$ is the phase speed of sound), the frequency dependent sound relaxational absorption spectrum $\alpha_{n} \lambda$ (i.e. the absorption coefficient per wavelength due to the $n$th single-relaxation process) is [17]

$$
\begin{gathered}
\alpha_{n} \lambda=2 \pi \cdot\left\{\left[\left(B_{n} / C_{n}\right)^{2}+1\right]^{1 / 2}-B_{n} / C_{n}\right\}, \\
B_{n}=x_{n}^{2}(\omega)+y_{n}^{2}(\omega)+R x_{n}(\omega), C_{n}=R y_{n}(\omega) .
\end{gathered}
$$

Here $x_{n}(\omega)=\operatorname{Re}\left(C_{n}^{\mathrm{eff}}\right)=C_{V}^{\infty}+\frac{C_{n}^{*}}{1+\left(\omega \tau_{n}\right)^{2}}$, and $y_{n}(\omega)=-\mathbf{I m}\left(C_{n}^{\mathrm{eff}}\right)=\frac{\omega \tau_{n} C_{n}^{*}}{1+\left(\omega \tau_{n}\right)^{2}}$.

In the works of Bauer [19,20], Bass[24] and Shields[25], they suppose that a multimode relaxation absorption spectrum can be decomposed into a sum of single-relaxation absorption spectra, each of them has a different relaxation time and relaxation strength. Thus, we assume that the relaxational absorption spectrum $\alpha_{\mathrm{r}} \lambda$ due to a multi-relaxation process can be decomposed into the sum of the spectra due to the decoupled single-relaxation processes characterized by $\tau_{n}(n=1,2, \mathrm{~L}, N)$ and $C_{n}^{*}(n=1,2, \mathrm{~L}, N)$, that is

$$
\alpha_{\mathrm{r}} \lambda=\sum_{n=1}^{N} \alpha_{n} \lambda
$$

\subsection{Vibrational mode-contribution for sound absorption}

The presence of relaxing gases is obviously manifested by relaxational peaks in the bell-shaped absorption spectrum [10,21]. The most important point in a single-relaxation absorption spectrum is its absorption maximum, the frequency and height of which are used to obtain the relaxation time and the relaxation strength respectively $[4,6]$. From Eq. (11), the minimum value of $B_{n} / C_{n}$ corresponds to the maximum height of $\alpha_{n} \lambda$. Thus, setting $\mathrm{d}\left(B_{n} / C_{n}\right) / \mathrm{d} \omega=0$, the frequency of absorption maximum (i.e. the relaxation frequency) for $\alpha_{n} \lambda$ is

$$
f_{n}^{\mathrm{m}}=\frac{1}{2 \pi \tau_{n}} \sqrt{\frac{\left(C_{V}^{\infty}+C_{n}^{*}\right)\left(C_{V}^{\infty}+C_{n}^{*}+R\right)}{C_{V}^{\infty}\left(C_{V}^{\infty}+R\right)}} .
$$

When $\omega=2 \pi f_{n}^{\mathrm{m}}$, the height of absorption maximum $\left(\alpha_{n} \lambda\right)_{\mathrm{m}}$ can be inferred from Eq. (13) as

$$
\left(\alpha_{n} \lambda\right)_{\mathrm{m}}=\frac{\pi}{2} \frac{\varepsilon_{n}}{\sqrt{\left(1-\varepsilon_{n}\right)}}, \quad \varepsilon_{n}=\frac{C_{n}^{*} R}{\left(C_{V}^{\infty}+C_{n}^{*}\right)\left(C_{V}^{\infty}+R\right)} .
$$

where $\varepsilon_{n}$ is the relaxation strength defined by Kneser [24]. It is generally true that $C_{n}^{*}<<C_{V}^{\infty}$ to make $\varepsilon_{n}<<1$ around room temperature [24]. Thus, we can rewrite $\left(\alpha_{n} \lambda\right)_{\mathrm{m}}$ in Eq. (14) as

$$
\left(\alpha_{n} \lambda\right)_{\mathrm{m}} \approx \frac{\pi}{2} \frac{C_{n}^{*} R}{C_{V}^{\infty}\left(C_{V}^{\infty}+R\right)} .
$$


Eq. (15) shows that the height of absorption maximum is proportional to the corresponding process IMH $C_{n}^{*}$, which means the bigger $C_{n}^{*}$ a single-relaxation process has, the stronger relaxation strength it possesses.

In our previous works [4], we have proven that a single-relaxation absorption spectrum $\alpha_{n} \lambda$ in the whole frequency range can be reconstructed from the point of absorption maximum, that is

$$
\alpha_{n} \lambda=\left(\alpha_{n} \lambda\right)_{\mathrm{m}} \frac{2 f_{n}^{\mathrm{m}} f}{f^{2}+\left(f_{n}^{\mathrm{m}}\right)^{2}} .
$$

Here $f$ is the frequency of sound. Eq. (16) shows that the absorption spectrum reaches a maximum when $f$ matches $f_{n}^{\mathrm{m}}$. Thus, for a single-relaxation absorption spectrum, the differences in the heights of absorption at different frequencies just come from the matching degrees between $f$ and $f_{n}^{\mathrm{m}}$ (or $\tau_{n}$ based on Eq. (13)). The matching degrees determine how much the acoustic energy retained by the vibrational modes will be converted to thermal energy during the relaxation transitions. Therefore, although the absolute contribution of a vibrational mode available in the mixture to the relaxational absorption is different at various frequencies in a single-relaxation absorption spectrum, the percent contribution will be the same at any frequency.

From Eqs. (15) and (16), we can see $\left(\alpha_{n} \lambda\right)_{\mathrm{m}}$ is linearly proportional to $C_{n}^{*}$ and the single-relaxation absorption spectrum $\alpha_{n} \lambda$ can be reconstructed from the point of absorption maximum; on the other hand, the vibrational modes only changes the value of $C_{n}^{*}$. Hence the percent contribution of vibrational modes to the absorption spectrum $\alpha_{n} \lambda$ is equivalent to $C_{n}^{*}$. Consequently, based on Eq. (7), we define $Z_{j, n}$ as the contribution of mode $j$ for $C_{n}^{*}$ or the $n$th single-relaxation process; that is

$$
Z_{j, n}=a_{j} C_{j}^{\mathrm{vib}} V_{j n} h_{n}^{\prime}, j, n=1,2, \mathrm{~L}, N
$$

Eq. (17) shows that $Z_{j, n}$ is decided by four factors: i ) The mole fraction $a_{j}$. ii) The vibrational IMH $C_{j}^{v i b}$, which is proportional to the ambient temperature and varies inversely with the characteristic frequency of mode $j[18]$. iii) The entry of the eigenvector matrix of the energy-transition-rate matrix $V_{j n}$, which is related to the $\mathrm{V}-\mathrm{V}$ and V-T energy transition rates between mode $j$ and the other modes. iv) $h_{n}^{\prime}$ is the outcome from the interactions among all modes, which is the same for all vibrational modes. In short, Eq. (17) demonstrates that the contribution of mode $j$ to the $n$th single-relaxation process is not just decided by itself but also dependent upon its interactions with the other modes. 
By combining Eqs. (7) and (17), the process IMH $C_{n}^{*}$ can be rewriten as $C_{n}^{*}=\sum_{j=1}^{N} Z_{j, n}$, demonstrating the IMH of a single-relaxation process is the sum of contributions from all vibrational modes, in other words, a relaxational absorption peak is related to all vibrational modes available to the gas. Further, the mode contribution $Z_{j, n}$ can be expressed in percentage as

$$
P_{j, n}=\left(Z_{j, n} / \sum_{j=1}^{N} Z_{j, n}\right) \times 100 \% \text {. }
$$

\section{Simulations and discussion}

\subsection{Decomposing multi-relaxation absorption spectrum}

To verify our model for decomposing the multi-relaxation absorption spectra in excitable gases, we consider the mixtures of $20 \% \mathrm{CH}_{4}-80 \% \mathrm{~N}_{2}, 40 \% \mathrm{CH}_{4}-60 \% \mathrm{~N}_{2}$, $60 \% \mathrm{CH}_{4}-40 \% \mathrm{~N}_{2}, \quad 80 \% \mathrm{CH}_{4}-20 \% \mathrm{~N}_{2}, \quad 20 \% \mathrm{CO}_{2}-80 \% \mathrm{~N}_{2}, \quad 40 \% \mathrm{CO}_{2}-60 \% \mathrm{~N}_{2}$, $60 \% \mathrm{CO}_{2}-40 \% \mathrm{~N}_{2}$ and $80 \% \mathrm{CO}_{2}-20 \% \mathrm{~N}_{2}$ at room temperature. In our previous works $[17,18]$, we directly calculated the multi-relaxation absorption by $\alpha_{\mathrm{r}} \lambda=-2 \pi \cdot \operatorname{Im}\left(c_{\mathrm{e}}^{-1}\right) / \operatorname{Re}\left(c_{\mathrm{e}}^{-1}\right)\left(c_{\mathrm{e}}=\sqrt{P_{0}\left(C_{V}^{\mathrm{eff}}+\mathrm{R}\right) /\left(\rho_{0} C_{V}^{\text {eff }}\right)}\right)$. In contrast, in this paper, we will obtain the multi-relaxation absorption by the sum of all single-relaxation absorption spectra, i.e. $\alpha_{\mathrm{r}} \lambda=\sum_{n=1}^{N} \alpha_{n} \lambda$ (Eq. (12)). $\mathrm{CH}_{4}$ has four vibrational modes, $\mathrm{CO}_{2}$ has three modes, and $\mathrm{N}_{2}$ has one mode [18]. So, for the mixture $\mathrm{CH}_{4}-\mathrm{N}_{2}$, its acoustic multi-relaxation process would contain five single-relaxation processes; for the mixture $\mathrm{CO}_{2}-\mathrm{N}_{2}$, four single-relaxation processes.. In Figures 1 and 2, the total acoustic absorption $\alpha \lambda$ (solid line) is the sum of the multi-relaxation absorption $\alpha_{\mathrm{r}} \lambda$ (dash line) calculated by Eq. (12) and the classical absorption $\alpha_{\mathrm{c}} \lambda$ (dotted line) associated with transport phenomena got from the Stokes and Kirchhoff formulation $[10,26]$. Comparisons show that the theoretical absorption spectra $\alpha \lambda$ agree fairly well with the experimental data (circles) from Ejakov et al [27]. 


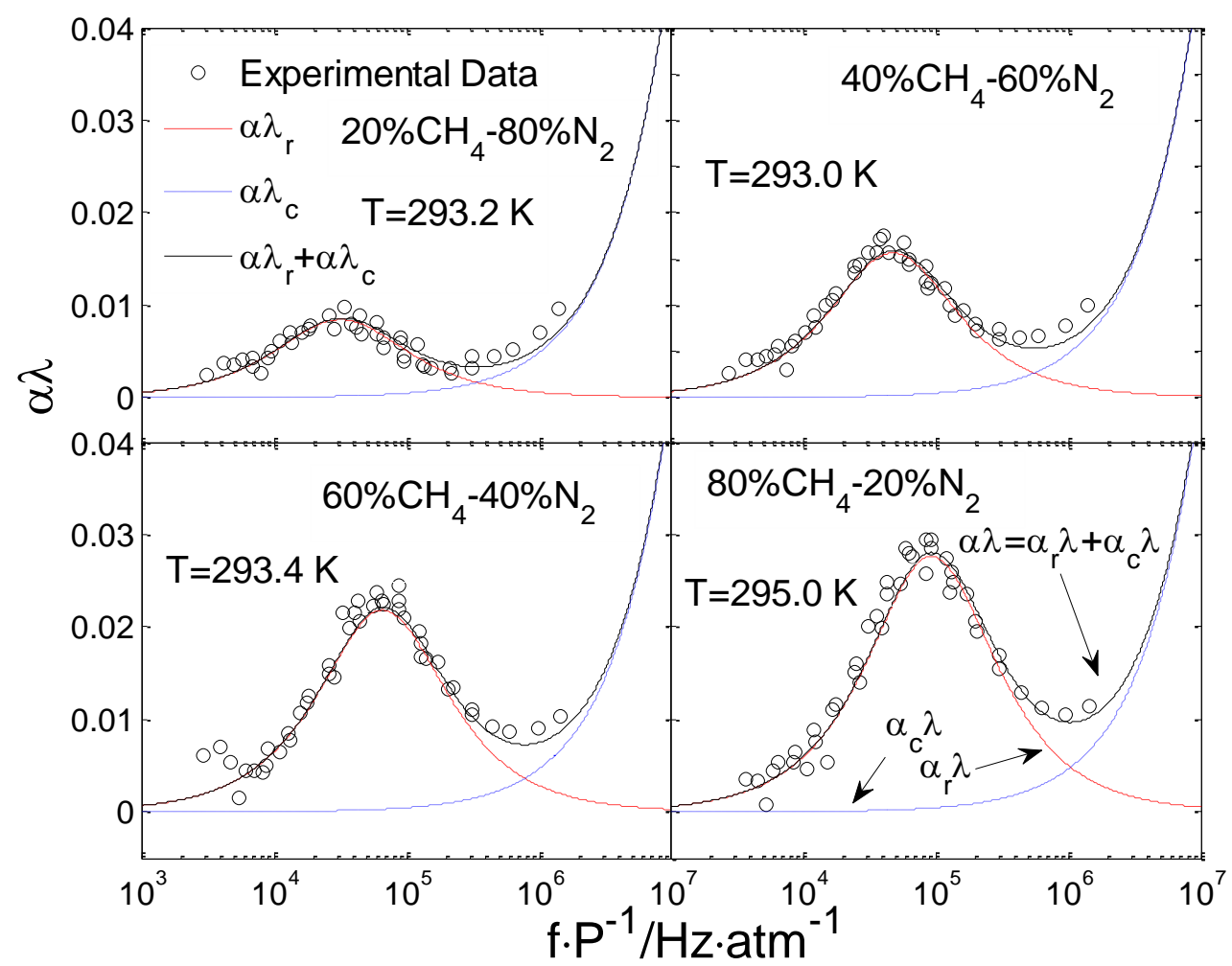

Fig. 1. Comparison of theoretical acoustic absorption spectra with experimental data from Ejakov et al. [27] for $\mathrm{CH}_{4}-\mathrm{N}_{2}$ mixtures around room temperature.

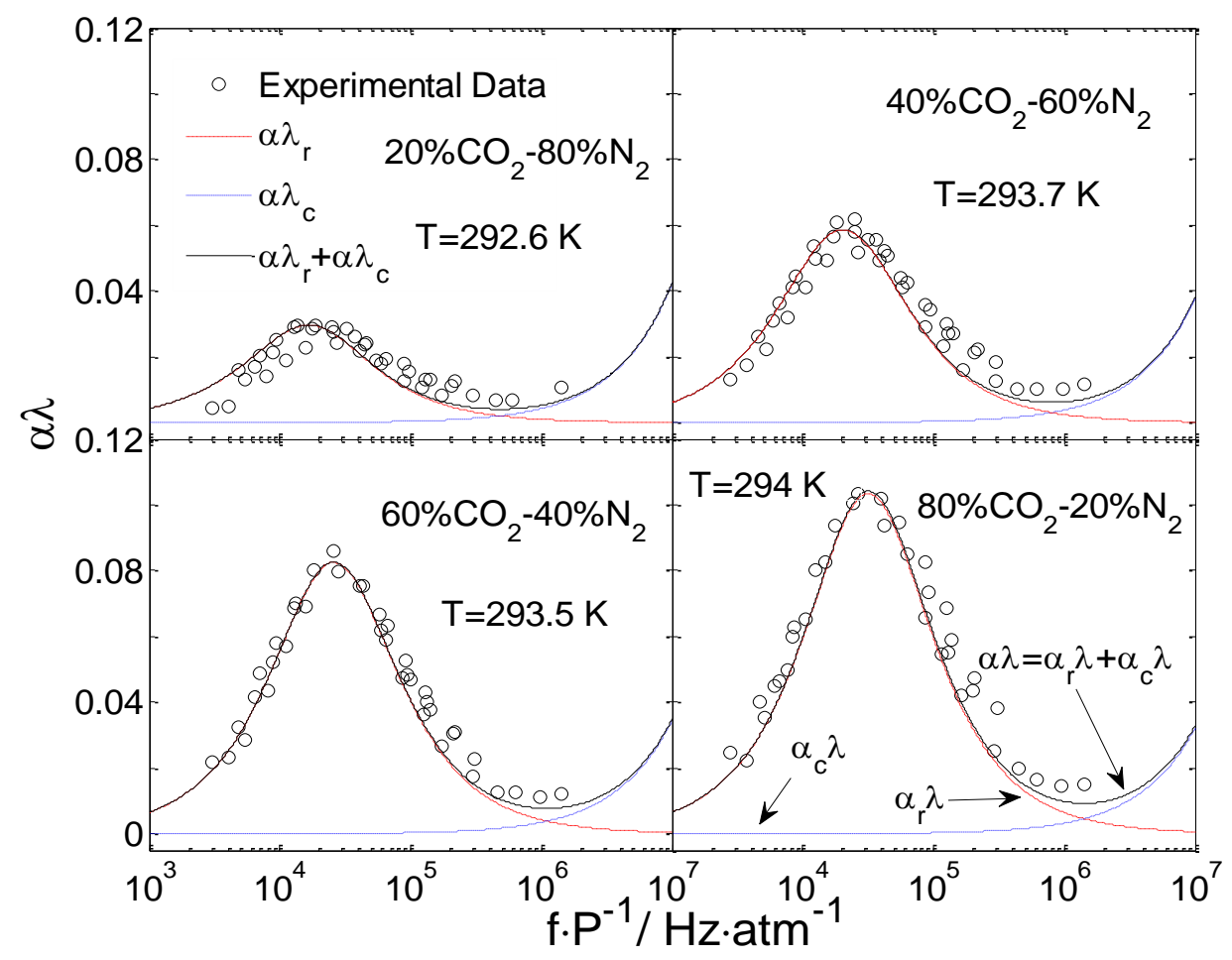

Fig. 2. Comparison of theoretical acoustic absorption spectra with experimental data from Ejakov et al. [27] for $\mathrm{CO}_{2}-\mathrm{N}_{2}$ mixtures around room temperature. 
The calculations of relaxation strengths by Eq. (14) and relaxation times by Eq. (7) (at $P=1 \mathrm{~atm}$ ) for all single-relaxation processes in the mixtures of Figs. 1 and 2 are given in Table 1. For $40 \% \mathrm{CH}_{4}-60 \% \mathrm{~N}_{2}, 60 \% \mathrm{CH}_{4}-40 \% \mathrm{~N}_{2}, 80 \% \mathrm{CH}_{4}-20 \% \mathrm{~N}_{2}$, $20 \% \mathrm{CO}_{2}-80 \% \mathrm{~N}_{2}, 60 \% \mathrm{CO}_{2}-40 \% \mathrm{~N}_{2}$ and $80 \% \mathrm{CO}_{2}-20 \% \mathrm{~N}_{2}$, it is found that only the relaxation strengths of 1 st processes are significant while the relaxation strengths of other processes are too small. So, the 1 st process is the primary relaxation process. As illustrated in Figures 3 and 4, if we just consider the influence of 1 st process to the acoustic propagation for each of those six mixtures, the 1 st single-relaxation absorption $\alpha_{1} \lambda$ calculated by Eq. (11) is overlapped with multi-relaxation absorption $\quad \alpha_{\mathrm{r}} \lambda \quad\left(40 \% \mathrm{CH}_{4}-60 \% \mathrm{~N}_{2}: \quad C_{1}^{*}=0.850 \mathrm{~J} \mathrm{~mol}^{-1} \mathrm{~K}^{-1} \quad ; \quad 60 \% \mathrm{CH}_{4}-40 \% \mathrm{~N}_{2}\right.$ : $C_{1}^{*}=1.29 \mathrm{~J} \mathrm{~mol}^{-1} \mathrm{~K}^{-1} \quad ; \quad 80 \% \mathrm{CH}_{4}-20 \% \mathrm{~N}_{2}: \quad C_{1}^{*}=1.76 \mathrm{~J} \mathrm{~mol}^{-1} \mathrm{~K}^{-1} \quad ; \quad 20 \% \mathrm{CO}_{2}-80 \% \mathrm{~N}_{2}$ : $C_{1}^{*}=1.43 \mathrm{~J} \mathrm{~mol}^{-1} \mathrm{~K}^{-1} \quad ; \quad 60 \% \mathrm{CO}_{2}-40 \% \mathrm{~N}_{2}: \quad C_{1}^{*}=4.33 \mathrm{~J} \mathrm{~mol}^{-1} \mathrm{~K}^{-1} \quad ; \quad 80 \% \mathrm{CO}_{2}-20 \% \mathrm{~N}_{2}$ : $C_{1}^{*}=5.96 \mathrm{~J} \mathrm{~mol}^{-1} \mathrm{~K}^{-1}$.). Therefore, although Eq. (12) shows that a multi-relaxation absorption spectrum can be decomposed into the sum of spectra due to the single-relaxation processes, whose number is the same as that of the vibrational modes available in the gas, the relaxation strengths of the most of these processes are so small that their influences to acoustic absorption can be ignored in practice.

Table 1. Calculations of the relaxation strengths and the relaxation times of single-relaxation processes at atmospheric pressure $(P=1 \mathrm{~atm}$ or $0.101325 \mathrm{MPa})$ in the gas mixtures of Figs. 1 and 2 .

\begin{tabular}{|c|c|c|c|c|c|c|c|c|c|c|}
\hline \multirow{2}{*}{ Gas } & \multicolumn{2}{|c|}{ 1st process } & \multicolumn{2}{|c|}{2 nd process } & \multicolumn{2}{|c|}{ 3rd process } & \multicolumn{2}{|c|}{ 4th process } & \multicolumn{2}{|c|}{5 th process } \\
\hline & $\varepsilon_{1}$ & $\tau_{1} / \mathrm{s}$ & $\varepsilon_{2}$ & $\tau_{2} / \mathrm{s}$ & $\varepsilon_{3}$ & $\tau_{3} / \mathrm{s}$ & $\varepsilon_{4}$ & $\tau_{4} / \mathrm{s}$ & $\varepsilon_{5}$ & $\tau_{5} / \mathrm{s}$ \\
\hline $20 \% \mathrm{CH}_{4}-80 \% \mathrm{~N}_{2}$ & $2.83^{-3}$ & $6.21^{-6}$ & $2.67^{-3}$ & $3.94^{-6}$ & $5.83^{-8}$ & $2.28^{-9}$ & $1.29^{-9}$ & $3.18^{-8}$ & $2.17^{-12}$ & $8.94^{-9}$ \\
\hline $40 \% \mathrm{CH}_{4}-60 \% \mathrm{~N}_{2}$ & $9.87^{-3}$ & $3.43^{-6}$ & $5.74^{-5}$ & $1.41^{-6}$ & $4.05^{-8}$ & $6.06^{-10}$ & $8.96^{-10}$ & $8.96^{-9}$ & $1.40^{-12}$ & $2.31^{-9}$ \\
\hline $60 \% \mathrm{CH}_{4}-40 \% \mathrm{~N}_{2}$ & $1.38^{-2}$ & $2.52-6$ & $3.72^{-8}$ & $2.88^{-10}$ & $1.72^{-8}$ & $7.12^{-7}$ & $8.64^{-10}$ & $4.48^{-9}$ & $9.48^{-13}$ & $1.06^{-9}$ \\
\hline $80 \% \mathrm{CH}_{4}-20 \% \mathrm{~N}_{2}$ & $1.74^{-2}$ & $1.82^{-6}$ & $2.96^{-6}$ & $4.27^{-7}$ & $3.94^{-8}$ & $1.69^{-10}$ & $9.68^{-10}$ & $2.70^{-9}$ & $8.07^{-13}$ & $6.06^{-10}$ \\
\hline $20 \% \mathrm{CO}_{2}-80 \% \mathrm{~N}_{2}$ & $1.85^{-2}$ & $9.93^{-6}$ & $7.76^{-4}$ & $5.74^{-5}$ & $5.36^{-5}$ & 1.01 & $3.91^{-12}$ & $1.86^{-6}$ & 1 & 1 \\
\hline $40 \% \mathrm{CO}_{2}-60 \% \mathrm{~N}_{2}$ & $2.84^{-2}$ & $7.66^{-6}$ & $9.45^{-3}$ & $1.18^{-5}$ & $4.42^{-5}$ & $1.61^{-1}$ & $1.53^{-11}$ & $2.80^{-6}$ & 1 & 1 \\
\hline $60 \% \mathrm{CO}_{2}-40 \% \mathrm{~N}_{2}$ & $4.92^{-2}$ & $7.38^{-6}$ & $2.32^{-3}$ & $3.97^{-6}$ & $8.48^{-10}$ & $3.11^{-6}$ & $4.26^{-5}$ & $7.79^{-2}$ & 1 & 1 \\
\hline $80 \% \mathrm{CO}_{2}-20 \% \mathrm{~N}_{2}$ & $6.37^{-2}$ & $6.26^{-6}$ & $6.11^{-4}$ & $2.20^{-6}$ & $4.25^{-5}$ & $8.80^{-2}$ & $4.92^{-9}$ & $2.47^{-7}$ & 1 & 1 \\
\hline
\end{tabular}
The notation $x^{y}$ implies $x \times 10^{y}$. 


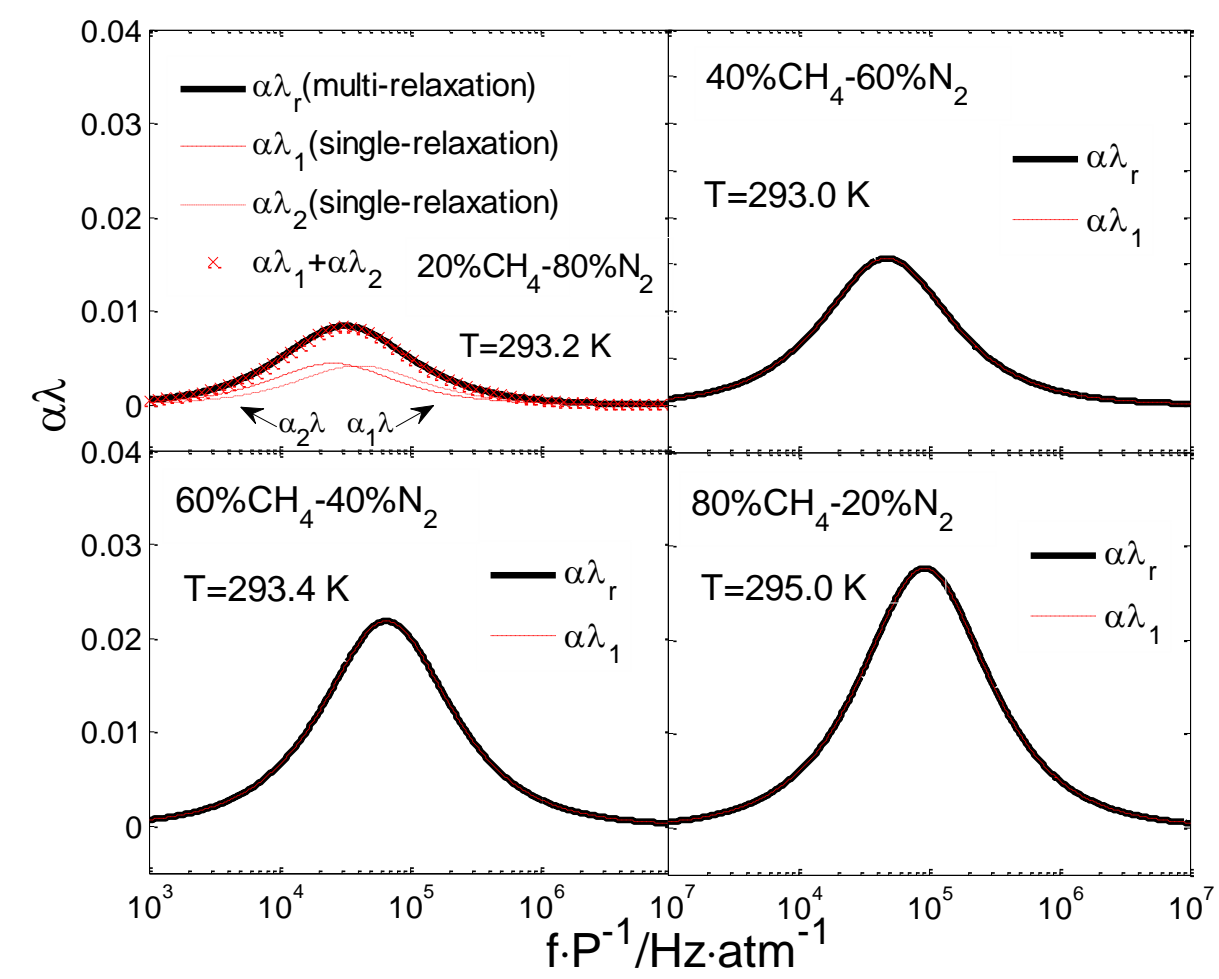

Fig. 3. Comparison of multi-relaxation absorption spectra with decomposed single-relaxation absorption spectra for $\mathrm{CH}_{4}-\mathrm{N}_{2}$ mixtures around room temperature. $\alpha_{\mathrm{r}} \lambda$ : multi-relaxation absorption; $\alpha_{1} \lambda: 1$ st single-relaxation absorption; $\alpha_{2} \lambda: 2$ nd single-relaxation absorption.

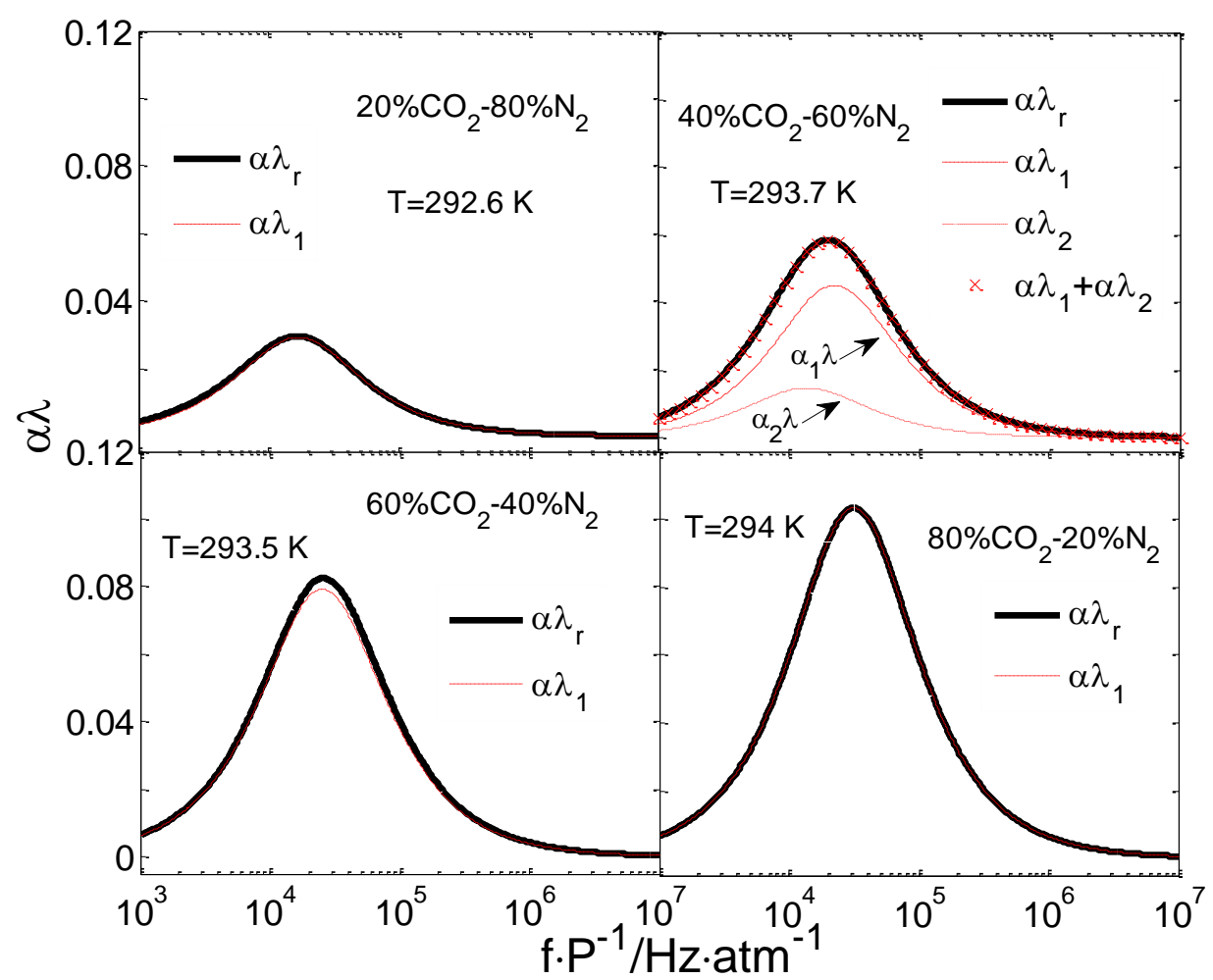

Fig. 4. Comparison of multi-relaxation absorption spectra with decomposed single-relaxation absorption spectra for $\mathrm{CO}_{2}-\mathrm{N}_{2}$ mixtures around room temperature. $\alpha_{\mathrm{r}} \lambda$ : the multi-relaxation absorption; $\alpha_{1} \lambda: 1$ st single-relaxation absorption; $\alpha_{2} \lambda: 2$ nd single-relaxation absorption. 
In conventional notion, one measurement absorption peak just corresponds to one single-relaxation process, i.e., if one absorption peak is measured, its corresponding single-relaxation process is found [10,19,21]. However, interestingly, although only one absorption peak is measured in the mixtures of $20 \% \mathrm{CH}_{4}-80 \% \mathrm{~N}_{2}$ and $40 \% \mathrm{CO}_{2}-60 \% \mathrm{~N}_{2}$, in fact there are two single-relaxation processes with significant relaxation strengths (i.e., the 1 st and 2 nd processes) shown in Table 1. For those two mixtures, the ultrasonic measurement cannot establish the maximum value of the absorption per wavelength for those two individual relaxation processes respectively. While based on our model, the two absorptions $\alpha_{1} \lambda$ and $\alpha_{2} \lambda$ of two single-relaxation processes can be found. The similar case that two relaxation processes contribute to one acoustic absorption peak is also reported by Beyer [28], who named this phenomenon as the "Double relaxation effects". From Figures 3 and 4 , the sum of 1 st single-relaxation absorption $\alpha_{1} \lambda$ and 2 nd single-relaxation absorption $\alpha_{2} \lambda$ is overlapped with $\alpha_{\mathrm{r}} \lambda \quad\left(20 \% \mathrm{CH}_{4}-80 \% \mathrm{~N}_{2}: C_{1}^{*}=1.43 \mathrm{~J} \mathrm{~mol}^{-1} \mathrm{~K}^{-1}\right.$, $C_{2}^{*}=0.0566 \mathrm{~J} \mathrm{~mol}^{-1} \mathrm{~K}^{-1} \quad ; \quad 40 \% \mathrm{CO}_{2}-60 \% \mathrm{~N}_{2}: \quad C_{1}^{*}=2.29 \mathrm{~J} \mathrm{~mol}^{-1} \mathrm{~K}^{-1} \quad$, $C_{2}^{*}=0.710 \mathrm{~J} \mathrm{~mol}^{-1} \mathrm{~K}^{-1}$.).

Consequently, not only the total acoustic absorption in excitable gases can be traditionally divided into two parts as classical and relaxational absorption, but also the relaxational absorption part under a multi-relaxation process can be further decomposed into the ones ascribed to the single-relaxation processes. Hence, for the expression of total acoustic absorption $\alpha \lambda=\alpha_{\mathrm{c}} \lambda+\alpha_{\mathrm{r}} \lambda[10]$ in excitable gases, it can be updated as

$$
\alpha \lambda=\alpha_{\mathrm{c}} \lambda+\sum_{n=1}^{N} \alpha_{n} \lambda
$$

\subsection{Vibrational mode contributions to acoustic absorption}

From Eq. (7), each $C_{n}^{*}$ contains parts of the mole-fraction heat capacities of all the vibrational modes available to the mixture, which is generated from the $\mathrm{V}-\mathrm{V}$ energy coupling formulated by the differential relaxation equations (Eq. (1)). On the other hand, each $\tau_{n}$ includes the lifetimes of all excited states which depend upon the transition probabilities of all V-T and V-V energy transitions. They mean that a multi-relaxation process is controlled by a strong V-V coupling, which influences both the relaxation frequencies and the relaxation strengths. Thus, every single-relaxation process relates with all vibrational modes. That is, for an acoustic multi-relaxation process, it would not be possible to allocate any single-relaxation process or decomposed single-relaxation absorption spectrum to a particular vibrational mode $[18,29]$. Moreover, an excitable gas can be treated as a mixture of its three kinds of DOF - translation, rotation, and vibration. In Eq. (9), the process effective IMH $C_{n}^{\text {eff }}$ 
has three elements $C_{V}^{\infty}, C_{n}^{*}$ and $\tau_{n}$. Translation and rotation affect the value of $C_{V}^{\infty}$, while vibration affects $C_{n}^{*}$ and $\tau_{n}$. Thus, based on Eqs. (13) and (14), we can find that the different parts (i.e. translation, rotation, and vibration) of a component molecule would have different (increasing or decreasing) effects on $f_{n}^{\mathrm{m}}$ and $\left(\alpha_{n} \lambda\right)_{\mathrm{m}}$. This indicates that a single-relaxation absorption spectrum is the result of complicated interactions among all DOF of the gas molecules. So, strictly speaking, it is just possible for us to assign a relaxational absorption spectrum to a relaxation process or the whole mixture rather than a gas component or a DOF. Fortunately, using the simplified expression of $\left(\alpha_{n} \lambda\right)_{\mathrm{m}}$ given by Eq. (15), we can calculate the contributions of vibrational modes to a single-relaxation process or the maximum height of absorption spectrum.

To analyze the contributions of vibrational modes to acoustical relaxational absorption, we have the following steps. Firstly, we calculate the vibrational mode contributions in pure gases $\mathrm{CH}_{4}(T=299.15 \mathrm{~K})$ and $\mathrm{CO}_{2}(T=296.15 \mathrm{~K})$. The four vibrational modes of $\mathrm{CH}_{4}$ lead to four single-relaxation processes (Table 2), but only the 1 st process has significant relaxation strength to cause an obvious acoustic absorption peak as measured by the experiments. Therefore, in this case, we can ignore the other non-primary relaxation processes, just analyze mode contributions to the 1 st process or the primary relaxation process $\left(\varepsilon_{1}=0.0215, C_{1}^{*}=2.34 \mathrm{~J} \mathrm{~mol}^{-1} \mathrm{~K}^{-1}\right)$.

Table 2. Calculations of the relaxation strengths and relaxation times of the four single-relaxation processes in pure $\mathrm{CH}_{4}(T=299.15 \mathrm{~K}, P=1 \mathrm{~atm})$.

\begin{tabular}{ccc}
\hline Process & $\varepsilon_{n}$ & $\tau_{n}(\mathrm{~s})$ \\
\hline lst & $2.15 \times 10^{-2}$ & $1.36 \times 10^{-6}$ \\
2nd & $4.44 \times 10^{-8}$ & $1.11 \times 10^{-10}$ \\
3rd & $1.22 \times 10^{-9}$ & $1.82 \times 10^{-9}$ \\
4th & $8.18 \times 10^{-13}$ & $3.93 \times 10^{-10}$ \\
\hline
\end{tabular}

Based on Eqs. (17) and (18), the vibrational mode contributions $Z_{j, 1}$ and $P_{j, 1}$ $(j=1,2, \ldots, 4)$ to the 1 st single-relaxation process in pure $\mathrm{CH}_{4}$ are given in Table 3 . The mode $v_{4}=1306$ provides about $79.2 \%$ contribution for the relaxational absorption, $v_{2}=1534$ contributes the remaining $20.8 \%$, while the contributions of $v_{1}=2915$ and $v_{3}=3019$ can be ignored. It is demonstrated that the contributions of modes decrease as the vibrational frequencies of the modes increase for pure $\mathrm{CH}_{4}$. This phenomenon can be explained by the fact that a vibrational mode with the lower frequency has the larger vibrational heat capacity to detained more acoustic energy. So, the more acoustic energy relaxed from this mode in the form of heat, the more weight on the contribution to sound absorption. 
Table 3. Vibrational mode contributions to the 1 st single-relaxation process (the primary relaxation process) in pure $\mathrm{CH}_{4}(T=299.15 \mathrm{~K})$.

\begin{tabular}{lcc}
\hline Modes $\left(\mathrm{cm}^{-1}\right)$ & $\begin{array}{c}Z_{j, 1} \\
\left(\mathrm{~J} \mathrm{~mol}^{-1} \mathrm{~K}^{-1}\right)\end{array}$ & $P_{j, 1}$ \\
\hline $\mathrm{CH}_{4}\left(v_{4}=1306\right)$ & 1.85 & $79.2 \%$ \\
$\mathrm{CH}_{4}\left(v_{2}=1534\right)$ & 0.486 & $20.8 \%$ \\
$\mathrm{CH}_{4}\left(v_{1}=2915\right)$ & $5.96 \times 10^{-4}$ & $\sim 0 \%$ \\
$\mathrm{CH}_{4}\left(v_{3}=3019\right)$ & $3.74 \times 10^{-4}$ & $\sim 0 \%$ \\
\hline
\end{tabular}

To further confirm our calculations on mode contributions for $\mathrm{CH}_{4}(T=299.15 \mathrm{~K})$, we compare the absorption spectra due to the primary relaxation processes when considering different vibrational modes. Selecting different vibrational modes will lead to different $C_{n}^{*}$ and $\tau_{n}$, these parameters will affect the frequency and the height of absorption maximum due to the primary relaxation process (as shown in Eqs. (13) and (15)). In Fig. 5, the height of relaxational absorption maximum is about 0.0360 given by the experimental data from Gravitt (crosses) and 0.0340 from Edmonds (circles) [15]. From our theoretical spectra (Solid), when considering the modes $v_{2}=1534$ and $v_{4}=1306$ together, the height is 0.0352 with less than $1 \%$ relative error compared with the average value (0.0350) of experimental data; only considering the mode $v_{2}=1534$, the height is 0.0076 ; for the case of mode $v_{4}=1306$, the height becomes 0.0276 . Therefore, the mode $v_{2}=1534$ provides about $21 \%$ contribution to the total height, and $v_{4}=1306$ supplies the remaining $79 \%$. The shifts of the relaxation frequencies (i.e., the frequency of absorption maximum) with different modes are resulted from the corresponding different relaxation times. The same results of mode contributions for the primary relaxation process have been obtained in Table 3. This result confirms that the mode contributions for the process IMH of a single-relaxation process are equivalent to those for the maximum height of absorption spectrum. 


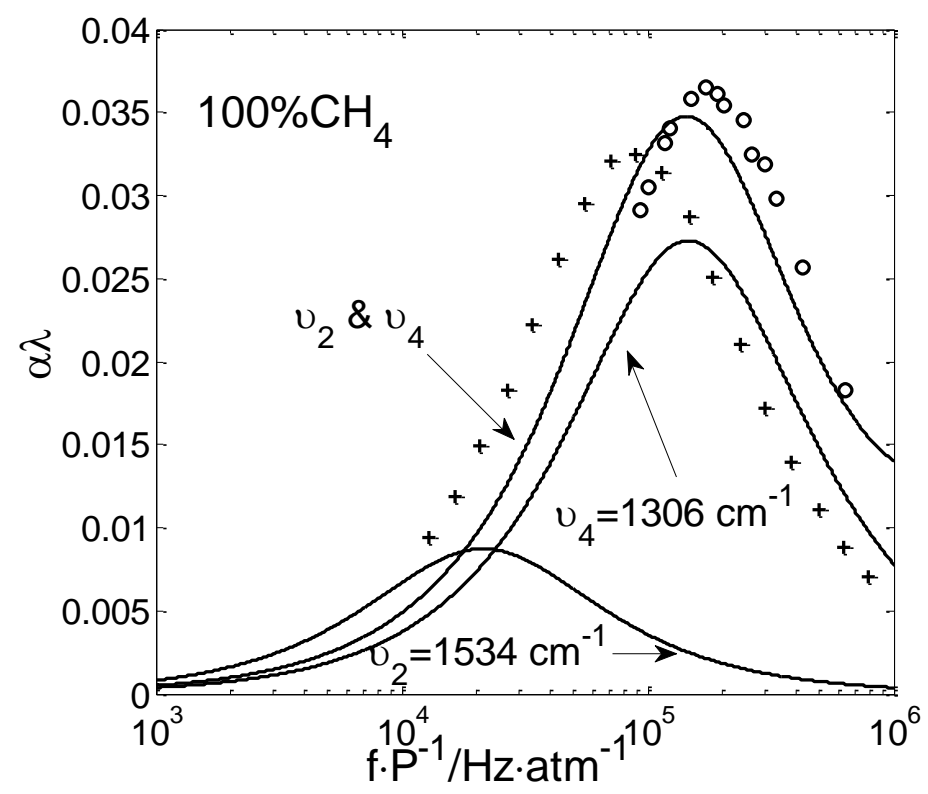

Fig. 5. Sound relaxational absorption for pure $\mathrm{CH}_{4}$. Symbols: Experiment data from Edmonds ( $T=298.15 \mathrm{~K}$, crosses), and Gravitt ( $T=299.15 \mathrm{~K}$, circles) [15]. Curves: theoretical absorption ( $T=299.15 \mathrm{~K}$, solid) due to the primary relaxation process, when considering the vibrational mode $v_{2}=1534 \mathrm{~cm}^{-1}, v_{4}=1306 \mathrm{~cm}^{-1}$, and $v_{2} \& v_{4}$, respectively (from lowest to highest).

Pure $\mathrm{CO}_{2}(T=296.15 \mathrm{~K})$ has three vibrational modes [18]. Table 4 provides the calculations of the corresponding relaxation strengths and relaxation times for three single-relaxation processes in the gas. Compared with the relaxation strength of $1 \mathrm{st}$ process $\left(\varepsilon_{1}=0.0763, C_{1}^{*}=7.57 \mathrm{~J} \mathrm{~mol}^{-1} \mathrm{~K}^{-1}\right)$, the relaxation strengths of the other two processes are so small that their effects on the absorption can be ignored. So we just need to analyze the mode contributions of the 1 st process (i.e., the primary process). In Table 5 , the bending mode $v_{2}=677$ contributes $96.2 \%$ to the primary process, providing an overwhelming contribution compared with other modes. Mode $v_{1}=1388$ gives $3.8 \%$ contribution, while the contribution of $v_{3}=2349$ is negligible. Therefore, the most decisive factor for the relaxational absorption in pure $\mathrm{CO}_{2}$ is the low-lying bending vibration of the molecule. This phenomenon can be explained by the fact that the energy gaps between the upper modes are usually much smaller than the energy level of the lowest mode which has the lowest characteristic frequency, so the rapid $\mathrm{V}-\mathrm{V}$ energy transitions maintain the continuous equilibrium of vibrational energy among all vibrational modes. Thus almost all resident acoustic energy relaxes through the $\mathrm{V}-\mathrm{T}$ deexcitation path provided by the lowest mode to arouse the primary relaxation process. This result also confirms that the mode with lower vibrational frequency holds more weight about the contribution to the primary relaxation process. 
Table 4. Calculations of the relaxation strengths and relaxation times of the three single-relaxation processes in pure $\mathrm{CO}_{2}(T=296.15 \mathrm{~K}, P=1 \mathrm{~atm})$.

\begin{tabular}{ccc}
\hline Process & $\varepsilon_{n}$ & $\tau_{n}(\mathrm{~s})$ \\
\hline 1st & $7.63 \times 10^{-2}$ & $5.01 \times 10^{-6}$ \\
2nd & $4.30 \times 10^{-4}$ & $1.45 \times 10^{-6}$ \\
3th & $4.47 \times 10^{-5}$ & $9.08 \times 10^{-2}$ \\
\hline
\end{tabular}

Table 5. Vibrational mode contributions to the 1 st single-relaxation process (the primary process) in pure $\mathrm{CO}_{2}(T=296.15 \mathrm{~K})$.

\begin{tabular}{ccc}
\hline Modes $\left(\mathrm{cm}^{-1}\right)$ & $\begin{array}{c}Z_{j, 1} \\
\left(\mathrm{~J} \mathrm{~mol}^{-1} \mathrm{~K}^{-1}\right)\end{array}$ & $P_{j, 1}$ \\
\hline $\mathrm{CO}_{2}\left(v_{2}=677\right)$ & 7.28 & $96.2 \%$ \\
$\mathrm{CO}_{2}\left(v_{1}=1388\right)$ & 0.29 & $3.8 \%$ \\
$\mathrm{CO}_{2}\left(v_{3}=2349\right)$ & $\sim 0$ & $\sim 0 \%$ \\
\hline
\end{tabular}

In Fig. 6, the relaxational absorption spectrum due to the primary relaxation process with $v_{2}=677$ (Dashed curve) is nearly overlapped with the one (Dotted line with cross markers) from the modes $v_{1}$ and $v_{2}$ or the one (Solid curve) from $v_{1}, v_{2}$ and $v_{3}$. These three theoretical spectra match with the experiment data from Kneser [24] (Circles) and Angona [30] (Squares) very well. These comparisons in $\mathrm{CO}_{2}$ confirm the correctness of our calculations on the vibrational mode contributions to acoustic relaxational absorption again.

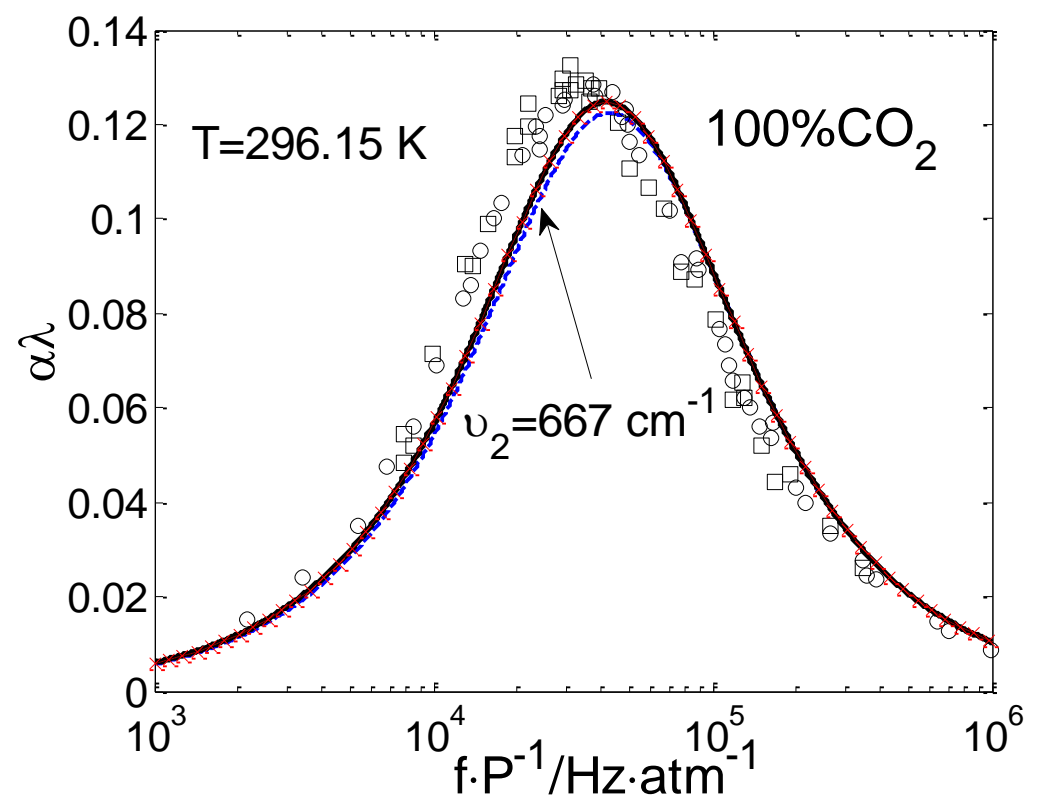

Fig. 6. Sound relaxational absorption for pure $\mathrm{CO}_{2}$. Symbols: Experiment data from Kneser [24] (Circles), and Angona [30] (Squares). Curves: Theoretical calculations from the primary relaxation process when considering the mode $v_{2}=667 \mathrm{~cm}^{-1}$ (Dashed), $v_{2} \& v_{1}=1388 \mathrm{~cm}^{-1}$ (Dotted line with cross markers) and $v_{1}, v_{2} \& v_{3}=2349 \mathrm{~cm}^{-1}$ (Solid), respectively. 
Secondly, we calculate the vibrational mode contributions in the two-component mixture of $5 \% \mathrm{CH}_{4}-95 \% \mathrm{~N}_{2}(T=300 \mathrm{~K})$. In Table 6 , we find that the relaxation strengths of 1 st and 2 nd single-relaxation processes are significant for the mixture. Hence, we just need consider the absorption spectra due to the 1 st and 2nd single-relaxation processes. In Fig. 7, the sum (dotted line with cross markers) of 1st single-relaxation absorption $\alpha_{1} \lambda$ (Dashed, $C_{1}^{*}=0.107 \mathrm{~J} \mathrm{~mol}^{-1} \mathrm{~K}^{-1}$ ) and 2 nd single-relaxation absorption $\alpha_{2} \lambda$ (Dash-dotted, $C_{2}^{*}=0.0188 \mathrm{~J} \mathrm{~mol}^{-1} \mathrm{~K}^{-1}$ ) is overlapped with multi-relaxation absorption $\alpha_{\mathrm{r}} \lambda$ (Solid). Thus, the multi-relaxation absorption $\alpha_{\mathrm{r}} \lambda$ can be decomposed by the sum of $\alpha_{1} \lambda$ and $\alpha_{2} \lambda$ with two obvious absorption peaks. Similarly, the two relaxational absorption peaks of $5 \% \mathrm{CH}_{4}-95 \% \mathrm{~N}_{2}$ are also predicted by DL model [3] (Dotted).

Table 6. Calculations of the relaxation strength and relaxation times of the five single-relaxation processes in the mixture of $5 \% \mathrm{CH}_{4}-95 \% \mathrm{~N}_{2}(T=300 \mathrm{~K}, P=1 \mathrm{~atm})$.

\begin{tabular}{ccc}
\hline Process & $\varepsilon_{n}$ & $\tau_{n}(\mathrm{~s})$ \\
\hline lst & $1.45 \times 10^{-3}$ & $4.75 \times 10^{-6}$ \\
2nd & $2.54 \times 10^{-4}$ & $8.61 \times 10^{-5}$ \\
3rd & $2.52 \times 10^{-7}$ & $3.62 \times 10^{-8}$ \\
4th & $1.09 \times 10^{-8}$ & $3.79 \times 10^{-7}$ \\
5th & $4.39 \times 10^{-11}$ & $1.31 \times 10^{-7}$ \\
\hline
\end{tabular}

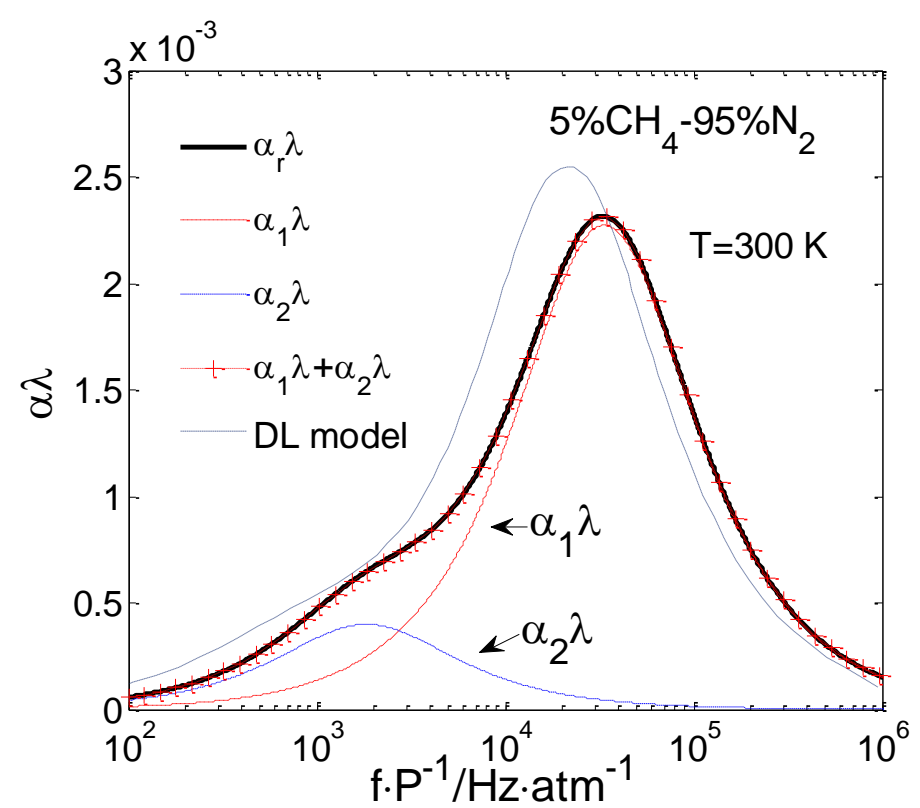

Fig. 7. Decomposing the sound multi-relaxation absorption spectrum in the mixture of $5 \% \mathrm{CH}_{4}-95 \% \mathrm{~N}_{2} . \quad \alpha_{\mathrm{r}} \lambda$ : multi-relaxation absorption curve; $\alpha_{1} \lambda: 1$ st single-relaxation absorption; $\alpha_{2} \lambda:$ 2nd single-relaxation absorption. 
We also give the calculated mode contributions for the 1 st and 2nd single-relaxation processes for the mixture of $5 \% \mathrm{CH}_{4}-95 \% \mathrm{~N}_{2}$. From Eq. (17), for a gas mixture, besides the vibrational frequency, the mole fraction of molecule and the other molecular natures (such as molecular weight, the depth of molecular potential well, collision diameter, etc.) both affect the mode-contribution in a mixture. In Table 7 , for the $1 \mathrm{st}$ process, the mode $\mathrm{CH}_{4}\left(v_{4}=1306\right)$ provides $79.5 \%$ contribution, $\mathrm{CH}_{4}\left(v_{2}=1534\right)$ makes $20.9 \%$, while the contributions of $\mathrm{CH}_{4}\left(v_{1}=2915\right)$ and $\mathrm{CH}_{4}\left(v_{3}=3019\right)$ are ignorable $(0.02 \%$ and $0.01 \%$ respectively $) . \mathrm{N}_{2}(v=2331)$ provides the negative value $-0.4 \%$, denoting that it is possible for a vibrational mode, which provides the $\mathrm{V}-\mathrm{T}$ deexcitation path for a single-relaxation process, to acquire slightly more energy in its $\mathrm{V}-\mathrm{V}$ energy exchanges than the relaxing energy by its $\mathrm{V}-\mathrm{T}$ transfer. For the mode contributions to the 2 nd process, $\mathrm{CH}_{4}\left(v_{4}=1306\right)$ is $44.8 \%, \mathrm{CH}_{4}\left(v_{2}=1534\right)$ is $11.8 \%, \mathrm{~N}_{2}(v=2331)$ is $43.4 \%$, the other two modes together provide negligible contributions (about $0.09 \%)$. The contributions of $\mathrm{CH}_{4}\left(v_{1}=2915\right)$ and $\mathrm{CH}_{4}\left(v_{3}=3019\right)$ for the absorption are so small that can be ignored in the prediction of sound relaxational absorption in practice. In this case, the mode contributions no longer decrease as the vibrational frequency increases.

Table 7. Vibrational mode contributions to 1 st and 2 nd single-relaxation processes in the mixture of $5 \% \mathrm{CH}_{4}-95 \% \mathrm{~N}_{2}(T=300 \mathrm{~K})$.

\begin{tabular}{ccccc}
\hline Modes $\left(\mathrm{cm}^{-1}\right)$ & $\begin{array}{c}Z_{j, 1} \\
\left(\mathrm{~J} \mathrm{~mol}^{-1} \mathrm{~K}^{-1}\right)\end{array}$ & $P_{j, 1}$ & $\begin{array}{c}Z_{j, 2} \\
\left(\mathrm{~J} \mathrm{~mol}^{-1} \mathrm{~K}^{-1}\right)\end{array}$ & $P_{j, 1}$ \\
\hline $\mathrm{CH}_{4}\left(v_{4}=1306\right)$ & $8.51 \times 10^{-2}$ & $79.5 \%$ & $8.41 \times 10^{-3}$ & $44.8 \%$ \\
$\mathrm{CH}_{4}\left(v_{2}=1534\right)$ & $2.24 \times 10^{-2}$ & $20.9 \%$ & $2.21 \times 10^{-3}$ & $11.8 \%$ \\
$\mathrm{CH}_{4}\left(v_{1}=2915\right)$ & $2.10 \times 10^{-5}$ & $\sim 0 \%$ & $1.15 \times 10^{-5}$ & $\sim 0 \%$ \\
$\mathrm{CH}_{4}\left(v_{3}=3019\right)$ & $1.41 \times 10^{-5}$ & $\sim 0 \%$ & $6.53 \times 10^{-6}$ & $\sim 0 \%$ \\
$\mathrm{~N}_{2}(v=2331)$ & $-4.49 \times 10^{-4}$ & $-0.4 \%$ & $8.15 \times 10^{-3}$ & $43.4 \%$ \\
\hline
\end{tabular}

Thirdly, we give the calculations of the vibrational mode contributions in the three-component mixture of $30 \% \mathrm{CL}_{2}-55.30 \% \mathrm{~N}_{2}-14.70 \% \mathrm{O}_{2}$ (i.e., $30 \% \mathrm{CL}_{2}-70 \%$ dry Air, $T=300 \mathrm{~K})$. For this mixture, only the relaxation strengths of 1 st single-relaxation processes are significant in Table 8; the 1st single-relaxation absorption $\alpha_{1} \lambda$ (dotted line with cross markers, $C_{1}^{*}=1.37 \mathrm{~J} \mathrm{~mol}^{-1} \mathrm{~K}^{-1}$ ) is overlapped with multi-relaxation absorption $\alpha_{\mathrm{r}} \lambda$ (Solid) in Fig. 8. In Table 9, $\mathrm{CL}_{2}(v=577)$ provides about $100 \%$ contribution for the 1 st process because of its quite low vibrational frequency, while the contributions of $\mathrm{N}_{2}(v=2331)$ and $\mathrm{O}_{2}(v=1554)$ are negligible (resulting in $\left.Z_{l, l}=C_{l}^{*}\right)$. 
Table 8. Calculations of the relaxation strength and relaxation times of the three single-relaxation processes in the mixture of $30 \% \mathrm{CL}_{2}-55.30 \% \mathrm{~N}_{2}-14.70 \% \mathrm{O}_{2}(T=300 \mathrm{~K}, P=1 \mathrm{~atm})$.

\begin{tabular}{ccc}
\hline Process & $\varepsilon_{n}$ & $\tau_{n}(\mathrm{~s})$ \\
\hline 1st & $1.76 \times 10^{-2}$ & $2.51 \times 10^{-6}$ \\
2nd & $2.01 \times 10^{-4}$ & $3.03 \times 10^{-3}$ \\
3rd & $5.31 \times 10^{-5}$ & $2.01 \times 10^{-2}$ \\
\hline
\end{tabular}

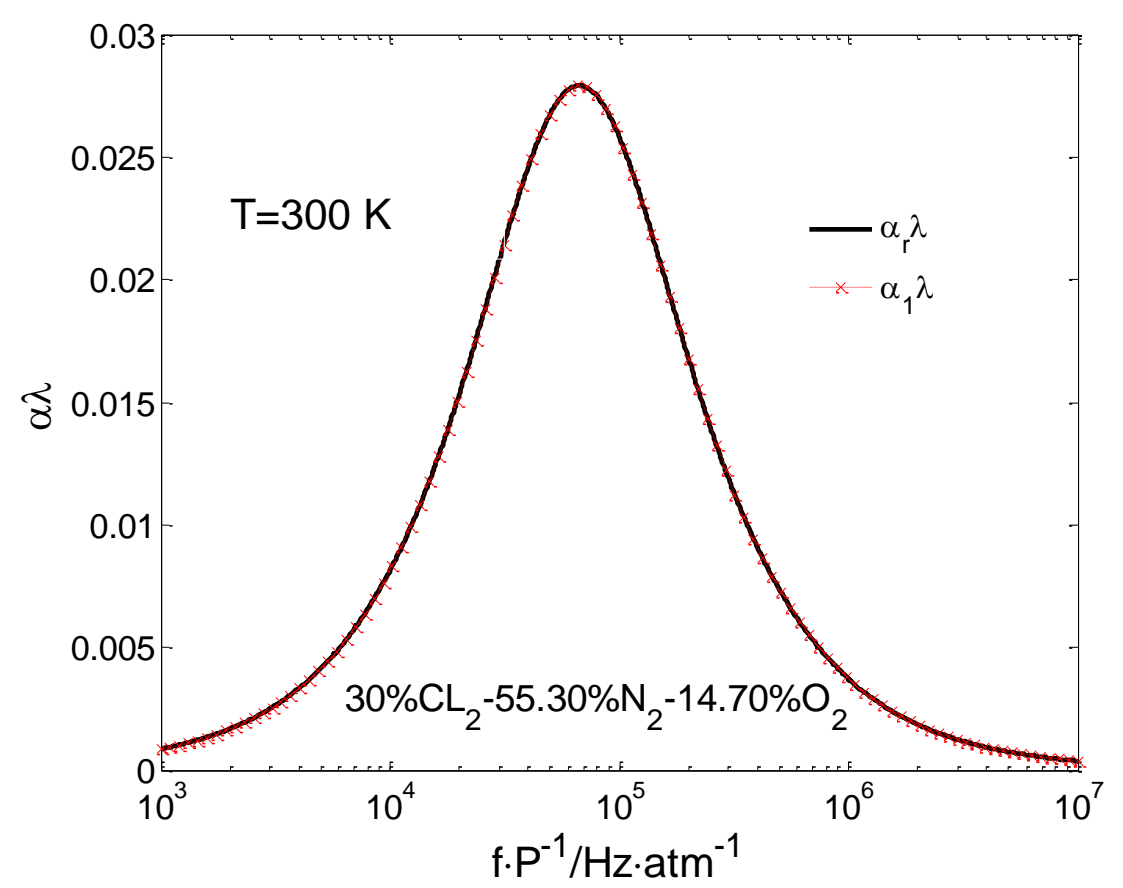

Fig. 8. Acoustic relaxational absorption in the mixture of $30 \% \mathrm{CL}_{2}-55.30 \% \mathrm{~N}_{2}-14.70 \% \mathrm{O}_{2} . \quad \alpha_{\mathrm{r}} \lambda$ : multi-relaxation absorption curve; $\alpha_{1} \lambda: 1$ st single-relaxation absorption.

Table 9. Vibrational mode contributions to the 1 st single-relaxation process in the mixture of $30 \% \mathrm{CL}_{2}-55.30 \% \mathrm{~N}_{2}-14.70 \% \mathrm{O}_{2}(T=300 \mathrm{~K})$.

\begin{tabular}{ccc}
\hline Modes $\left(\mathrm{cm}^{-1}\right)$ & $\begin{array}{c}Z_{j, 1} \\
\left(\mathrm{~J} \mathrm{~mol}^{-1} \mathrm{~K}^{-1}\right)\end{array}$ & $P_{j, 1}$ \\
\hline $\mathrm{CL}_{2}(v=577)$ & 1.37 & $\sim 100 \%$ \\
$\mathrm{~N}_{2}(v=2331)$ & $1.01 \times 10^{-11}$ & $\sim 0$ \\
$\mathrm{O}_{2}(v=1554)$ & $-9.56 \times 10^{-6}$ & $\sim 0$ \\
\hline
\end{tabular}

\section{Conclusions}

In this paper, we demonstrate how a sound multi-relaxation absorption spectrum is decomposed into the sum of single-relaxation processes spectra. Based on this decomposable characteristic, we calculate the vibrational mode contributions to the 
sound relaxational absorption in excitable gas mixtures at room temperature.

We show that not only the total sound absorption in excitable gases can be traditionally divided into two parts as classical and relaxational absorption, but also a multi-relaxation absorption can be further decomposed into the ones ascribed to the single-relaxation processes. We prove that the mode contributions for the process IMH of a single-relaxation process are equivalent to those for the maximum height of absorption spectrum. In most excitable gases, the primary relaxation process plays a decisive role in arousing the sound relaxational absorption and the mode with lower vibrational frequency provides higher contribution to the primary relaxation process in pure polyatomic gases. For pure $\mathrm{CH}_{4}$, the vibrational mode $v_{4}=1306$ has $79.2 \%$ contribution to the primary process, $v_{2}=1534$ provides $20.8 \%$ contribution, and the contributions of $v_{1}=2915$ and $v_{3}=3019$ are ignorable. For $\mathrm{CO}_{2}$, the bending mode $v_{2}=677$ has an overwhelming weight, $96.2 \%$ contribution, for the primary process; the mode $v_{1}=1388$ gives $3.8 \%$ and the contribution of $v_{3}=2349$ is negligible. For a gas mixture, besides the vibrational frequency, the mole fraction of molecule and the other molecular natures (such as molecular weight, the depth of molecular potential well, collision diameter, etc.) would jointly affect the mode-contribution. In short, our work provides an effective model to decompose a sound multi-relaxation absorption spectrum and calculate the vibrational mode contributions to sound relaxational absorption. Additionally, we find that in some situations such as in the mixtures of $20 \% \mathrm{CH}_{4}-80 \% \mathrm{~N}_{2}$ and $40 \% \mathrm{CO}_{2}-60 \% \mathrm{~N}_{2}$, there are two significant single-relaxation processes affecting the sound absorption, although only one absorption peak or a single-relaxation process can be measured by using acoustic approach.

Since the approach based on acoustic absorption spectra has been employed in moderate-sensitivity gas composition sensing [1-8], the quantitative relation between the molecular makeup of gas mixtures and acoustic absorption revealed in this paper can be used to guide the implementation of gas detection with acoustic absorption spectra.

\section{Acknowledgment}

We are very grateful to Professor Richard M. Lueptow for helpful advices. This work is supported by the National Natural Science Foundation of China (Grant Nos. 61461008, 61371139, 61571201, 61540051 and 51567017), the National Science Foundation of Guizhou Province, China (Grant No. [2015]2065), and the Recruitment Program of Guizhou Institute of Technology (Grant No. XJGC20140601).

\section{References}

[1] Phillips S, Dain Y, Lueptow RM. Theory for a gas composition sensor based on acoustic properties. Meas Sci Technol 2003; 14:70-5.

[2] Zhu M, Wang S, Wang ST, Xia DH. An acoustic gas concentration measurement algorithm for carbon monoxide in mixtures based on molecular multi-relaxation model. Acta Phys Sin 2008; 
57(9): 5749

[3] Petculescu AG, Lueptow RM. Synthesizing primary molecular relaxation processes in excitable gases using a two-frequency reconstructive algorithm. Phys Rev Lett 2005; 94: 238301

[4] Zhang KS, Wang S, Zhu M, Ding Y. Algorithm for capturing primary relaxation processes in excitable gases by two-frequency acoustic measurements. Meas Sci Technol 2013; 24: 055002

[5] Petculescu A, Lueptow R M. Quantitative Acoustic Relaxational Spectroscopy for real-time monitoring of natural gas: A perspective on its potential. Sensors \& Actuators B: Chemical 2012; 169: 121-7.

[6] Hu Y, Wang S, Zhu M, Zhang KS, Liu TT, Xu DY. Acoustic absorption spectral peak location for gas detection. Sensors \& Actuators B: Chemical 2014; 203: 1-8

[7] Zhang KS, Chen LK, Ou WH, Jiang XQ, Long F. A theory for monitoring combustion of natural gas based on the maximum point in sound absorption spectrum. Acta Phys Sin 2015; 64: 054302 .

[8]Hu Y, Wang S, Zhu M. A relaxation times coupling method to construct acoustic relaxation calibration for two-frequency measuring gas compositions. Applied Acoustics 2016; 113: 102-8.

[9] Mason WP, Thurston RN. Physical Acoustics (Vol. XVI). New York: Academic; 1984. p. 1-35.

[10] Herzfeld KF, Litovitz TA. Absorption and Dispersion of Ultrasonic Waves. New York: Academic; 1959. p. 55-216.

[11] Herzfeld KF, Rice FO. Dispersion and Absorption of High Frequency Sound Waves. phys rev 1928; 31 (4): 691-5.

[12] Landau L, Teller E. Zur theorie der Schalldispersion. Phys Z Sowjetunion 1936; 10 (1): 34 43.

[13] Schwartz RN, Slawsky ZI, Herzfeld KF. Calculation of vibrational relaxation times in gases. J Chem Phys 1952; 20 (10): 1591-1600

[14] Tanczos FI. Calculation of vibrational relaxation times of the chloromethanes. J Chem Phys 1956; 25 (3):439-447

[15] Dain Y, Lueptow RM. Acoustic attenuation in three-component gas mixtures-Theory. J Acoust Soc Am 2001; 109 (5): 1955-64

[16] Petculescu AG, Lueptow RM. sensitivity of the predicted attenuation to the Lennard-Jones parameters. J Acoust Soc Am 2005; 117 (1): 175-84

[17] Zhang KS, Wang S, Zhu M, Hu Y, Jia YQ. Analytical model for acoustic multi-relaxation spectrum in gas mixtures. Acta Phys Sin 2012; 61 (17): 174301

[18] Zhang KS, Wang S, Zhu M, Ding Y, Hu Y. Decoupling multimode vibrational relaxations in multi-component gas mixtures: analysis of sound relaxational absorption spectra. Chin Phys B 2013; 22 (1): 014305

[19] Mason WP, Thurston RN. Physical Acoustics (Vol. XVII) Orlando: Academic; 1984. p. 145232.

[20] Bauer HJ, Shields FD, Bass HE. Multimode Vibrational Relaxation in Polyatomic Molecules. J Chem Phys 1972; 57 (11): 4624-8

[21] Lambert JD. Vibrational and Rotational Relaxation in Gases. Oxford: Clarendon; 1977.

[22] Morse PM, Ingard KU. Theoretical acoustics. New York: McGraw-Hill; 1968.

[23] Zhang KS, Zhu M, Tang W Y, Ou WH, Jiang XQ. Algorithm for reconstructing vibrational relaxation times in excitable gases by two-frequency acoustic measurements. Acta Phys Sin 2016; 65(13): 134302 
[24] Mason WP. Physical Acoustics (Vol. 2). New York: Academic; 1965. p. 47-199

[25] Shields FD, Warf CC, Bass HE. Acoustical method of obtaining vibrational transition rates tested on CO2/N2 mixtures. J Chem Phys 1973; 58 (9): 3837-40

[26] Petculescu A, Achi P. A model for the vertical sound speed and absorption profiles in Titan's atmosphere based on Cassini-Huygens data. J Acoust Soc Am 2012; 131(5): 3671-9

[27] Ejakov SG, Phillips S, Dain Y, Lueptow RM, Visser JH. Acoustic attenuation in gas mixtures with nitrogen: Experimental data and calculations. J Acoust Soc Am 2003; 113(4): 1871-9.

[28] Beyer RT. Double relaxation effects. J Acoust Soc Am 1957; 29: 243-248

[29] Zuckerwar AJ, Miller KW. Vibrational-Vibrational coupling in air at low humidities. J Acoust Soc Am 1988; 84(3): 970-7

[30] Angona FA. The absorption of sound in gas mixtures. J Acoust Soc Am 1953; 25(6): 1116-22. 